\title{
Acesso lexical na afasia
}

\section{Lexical access in aphasia}

\section{Fernanda Duarte Senna ${ }^{1}$, Christina Abreu Gomes²}

Doutora em Linguística pela Universidade Federal do Rio de Janeiro. Fonoaudióloga pela Universidade Federal do Rio de Janeiro.

E-mail: fonofernandasenna@gmail.com Professora Titular da Universidade Federal do Ri॰ de Janeiro, Doutora em Linguística pela UFRJ e estágio pos-doutoral na University or Bolsista de Produlividade do CNPq.
RESUMO: 0 presente artigo teve como objetivo analisar o acesso lexical em afásicos e controles. Os dados foram obtidos através de um Teste de Nomeação de figuras (SNODGRASS e VANDERWARTS, 1980). Os pressupostos dos Modelos baseados no Uso foram adotados na análise dos dados no que diz respeito à organização do léxico em redes baseadas em similaridades sonoras e semânticas entre os itens lexicais (BYBEE, 1995, 2010), assumindo-se que as falhas no acesso lexical podem ser explicadas pelo modelo de redes. Também se adotou a hipótese da continuidade entre população típica e atípica no acesso lexical (DELL et al., 1997). Os resultados demonstraram que afásicos e controles diferem quantitativamente, mas não qualitativamente, em relação aos tipos de respostas das nomeações. Também ficou demonstrada a importância das variáveis idade de aquisição e familiaridade dos itens lexicais para os dois grupos estudados e da frequência de ocorrência do item lexical somente para os afásicos no desempenho na tarefa de nomeação.

Palavras-chave: Afasia; Acesso lexical; Anomia, Modelos baseados no Uso.

ABSTRACT: The present paper aimed to analyze the lexical access in aphasic and control subjects. Data were obtained using a Naming Picture Test (SNODGRASS \& VANDERWARTS, 1980). The theoretical assumptions of the Usage-based Models were adopted for data analysis in which it concerns the lexicon organization in networks based on sound and meaning similarities among the lexical items (BYBEE, 1995, 2010), assuming that failure in lexical access can be explained by the network model (BYBEE, 1995). It was also adopted the continuity hypothesis between typical and atypical population in lexical access (DELL et al., 1997). The results showed that aphasics and controls differ quantitatively, but not qualitatively, in relation to the type of responses in naming. It was also shown the importance of the variables age of acquisition and familiarity to lexical items for both studied groups and that of the token frequency of the lexical items only for the aphasics in the performance in the naming task.

Keywords: Aphasia; Lexical access; Anomie; Usage-based Models. 


\section{Introdução}

s estudos sobre acesso lexical no português brasileiro são poucos, seja com população típica, ou seja, com desenvolvimento dentro dos padrões esperados para sua comunidade de fala e sem alterações decorrentes de comprometimento cerebral ou de outra natureza (FRANÇA et al., 2008; ALVES, 2010; MEDEIROS et al., 2014), seja em afásicos (LIMA, 2010; FEIDEN, 2014), isto é, que apresentam comprometimento linguístico em função de lesão no cérebro. A possibilidade de analisar os dados sob uma nova ótica, a dos Modelos baseados no Uso, adotada na pesquisa, pode trazer novas contribuições para o entendimento do acesso lexical tanto em população típica quanto em população atípica, assim como para as questões teóricas tratadas no âmbito dessa teoria, além de contribuições para a clínica fonoaudiológica. Segundo Dell et al. (1997:801), tomando como base Freud (1891/1953, apud DELL et al., op. cit), as falhas nos mecanismos de acesso lexical em afásicos e indivíduos típicos devem ser entendidas como diferindo apenas em graus diferentes de comprometimento, não na sua natureza.

A afasia se apresenta de forma bastante variada, já que, em cada tipo de afasia, há predomínio de diferentes tipos de manifestação linguística. Essas manifestações têm sido classificadas sob os seguintes rótulos: a) anomia: falha na nomeação; ecolalia: repetição de parte da fala do outro; (c) agramatismo: falhas na relação gramatical da organização frasal; (d) jargão: fala incompreensível; (e) perseveração: repetição de uma palavra, geralmente a última a ser produzida; (f) confabulação: fala interior com perda de foco; (g) estereotipia: repetição de uma palavra, expressão ou segmento fonológico; (h) digressão: fala emendada perdendo o foco, entre outras. No entanto, toda afasia tem algum comprometimento na produção verbal, mesmo que seja em decorrência de dificuldades de compreensão.
Falha de acesso lexical ou anomia ${ }^{1}$ é a manifestação linguística mais comum presente no discurso oral de indivíduos com quase todos os tipos de afasia. A falha no acesso lexical se manifesta de diversas maneiras no discurso dos afásicos seja através de vazio no discurso, substituições de itens lexicais que podem envolver criação de novas palavras na língua a partir dos morfemas disponíveis (neologismos) ou de novas formas sonoras (pseudopalavras), repetição de um mesmo ato motor (perseveração), uso de termos genéricos, como coisa, negócio, troço, e, ainda, substituições que envolvem, por exemplo, semelhança semântica ou sonora entre as palavras, classificadas como parafasias, como, respectivamente, joelho por tornozelo, trapo por trato, ou substituições aleatórias como açúcar por leão.

É objetivo deste artigo apresentar resultados de estudo sobre acesso lexical em indivíduos afásicos (SENNA, 2013) a partir do desempenho em Teste de Nomeação. As questões e hipóteses de trabalho se baseiam nos pressupostos de organização do léxico dos Modelos baseados no Uso, segundo os quais os itens lexicais são representados no léxico mental em redes de relações lexicais baseadas em semelhanças sonoras, semânticas ou ambas. As representações lexicais são detalhadas e a experiência com o uso tem impacto na representação (BYBEE, 1995; 2010).

As seções a seguir apresentam os pressupostos teóricos que embasaram a pesquisa, questões de trabalho e metodologia, análise dos dados e considerações finais.

\section{Pressupostos teóricos}

Segundo Ortiz (2010), a afasia pode ser definida como uma alteração no conteúdo, na forma e no uso da linguagem e em seus processos cognitivos

${ }^{1} \mathrm{O}$ termo anomia tem sido utilizado como referência às falhas de acesso lexical e foi mantido neste artigo em consonância com a literatura da área. 
subjacentes, tais como percepção e memória. Segundo Mansur e Machado (2004), a afasia é definida como alteração da comunicação adquirida em consequência de lesão neurológica, em geral acidente vascular encefálico (AVE), e não de déficits sensoriais, intelectuais ou psiquiátricos. Envolve as modalidades de produção e compreensão das linguagens oral e escrita.

As primeiras divisões dos tipos de afásicos se basearam na dicotomia distúrbios expressivos, lesões localizadas em áreas mais anteriores do cérebro,e receptivos, lesões localizadas em áreas mais posteriores, caracterizando uma abordagem localizacionista, desenvolvida século XIX, por Paul Broca (1824-1880) e Karl Wernicke (1848-1905)(cf. GANDOLFO, 2006). Já a classificação clinica tradicional se fundamenta em uma série de variáveis fundamentais como linguagem espontânea, compreensão, repetição e nomeação, conforme Figura 1 a seguir.

Figura 1 - Esquema do modelo clássico de classificações das afasias de BENSON e ARDILA (1996), adaptado por Drummond (2006)

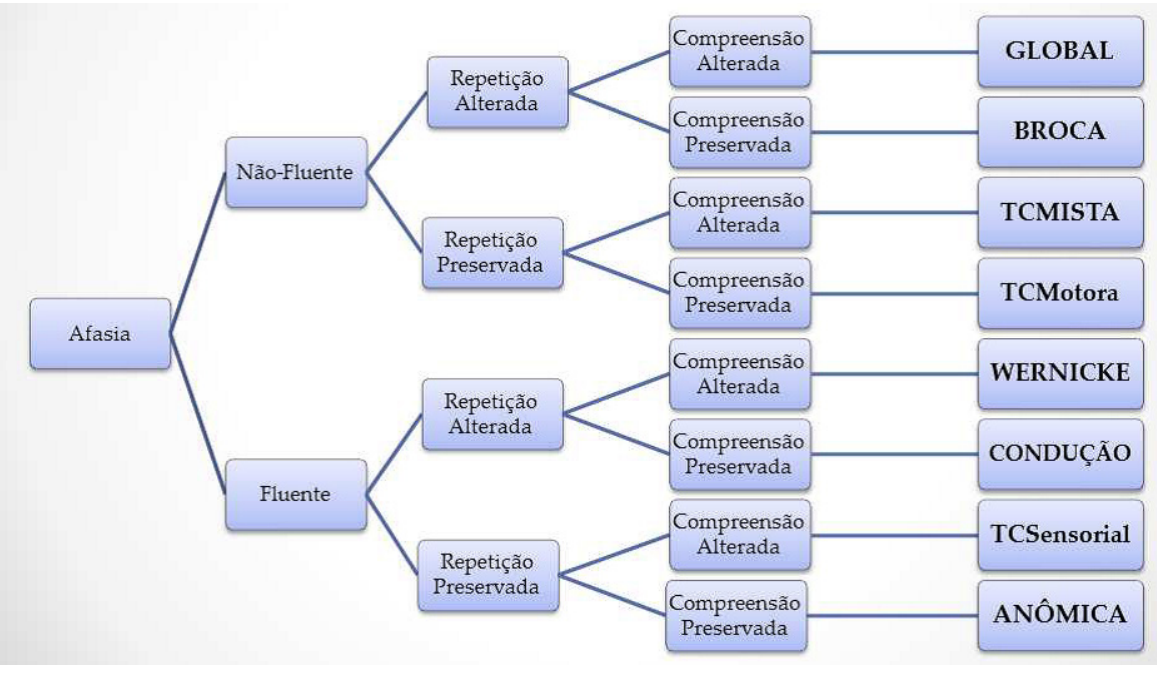

Fonte: SENNA (2013, p. 39).
Para Cuetos (2003) e Cuetos et al. (2010), não há relação direta entre local de lesão e a manifestação linguística. Assim como a localização das funções no cérebro, as lesões também não são rigorosamente delimitadas ou pontuais, ratificando a dificuldade em prever fielmente a sintomatologia a partir da lesão, ou vice versa. Assim a visão localizacionista vai dando lugar à abordagem conexionista, que parte do princípio de uma representação cerebral por centros conectados, e que, devido à lesão cerebral, o sujeito perde a capacidade de estabelecer a relação entre um centro e suas conexões, conexões essas que são modificadas pelo uso linguístico, por estímulo, associações, etc (CAPLAN, 1987; GANDOLFO, 2006). Ortiz (2010) aponta a dificuldade em definir as manifestações afásicas, baseando-se em classificações, e em agrupá-las em tipos sindrômicos. Além disso, destaca que, mais do que classificar, é fundamental esclarecer o distúrbio de linguagem e traçar diretrizes para a reabilitação, compreender o modo e o porquê das manifestações. Coudry (1988) defende que, na abordagem da afasia, seja levada em conta a relação do sujeito com a cultura e história.

Ortiz (2010) defende a relação da alteração de compreensão com dificuldades de emissão, dizendo que, mesmo que não haja aparentemente dificuldade de compreensão, há falhas no processamento das informações recebidas na comparação com sujeitos normais, dificuldades essas detectadas apenas por avaliação minuciosa. A anomia é definida como dificuldade de recordar ou recuperar as palavras e se manifesta na produção lingüística através de hesitações, substituições, etc. Segundo a autora, os processos de busca e recuperação das palavras são os mesmos para afásicos e não afásicos, mas, em pessoas com lesão, a operação se torna lenta, trabalhosa e muitas vezes não efetiva.

Dell, Schwartz, Martin, Saffran e Gagnon (1997) adotam a teoria da globalidade, que defende que as lesões acabam por afetar uniformemente todos os níveis do processamento lexical, bem como as interações entre eles, 
e que as diferentes manifestações na produção dos afásicos se relacionam a graus diferentes de comprometimento dos indivíduos e também a interações entre os níveis. No estudo de Dell et al. (1997), com afásicos fluentes (Afasia Anômica, Wernicke, de Condução ou Transcortical Sensorial), a produção dos afásicos foi observada a partir de um Teste de Nomeação. Foi considerada apenas a primeira resposta e os erros ou substituições foram classificados em semântico, fonológico, misto, pseudopalavra e sem resposta. Com esses critérios e baseados na teoria da globalidade, elaboraram um modelo computacional a fim de prever as distribuições dos erros. Os autores propõem um modelo considerando redes lexicais e o acesso lexical envolvendo duas etapas, acesso à informação conceptual e à informação sonora, e a interação entre elas. Como se trata de uma relação arbitrária entre forma e significado, motiva o surgimento de uma etapa intermediária de mapeamento, que nada mais é do que a interação entre as duas outras etapas. As duas fases distintas no acesso lexical permitem fluxo top-down e bottom-up(unidades semânticas ou fonológicas primeiramente ativadas no acesso lexical). Entre afásicos foi constatada uma considerável variação entre os indivíduos, de $8 \%$ a 95\% de acerto. Em alguns casos, houve predomínio de neologismos, em outros houve predomínio de substituições semânticas e, em outros, de substituições formais, referentes à substituição de sons. Dell et al. (1997) observaram a influência da categoria sintática nos erros formais. Embora afásicos e controles apresentem parafasias semânticas, no caso dos afásicos, não houve predomínio exclusivo de substituição semântica. Ainda, nas afasias mais graves, observou-se a presença, em maior número, de pseudopalavras e palavras não relacionadas com o alvo, o que reflete erros com discordância entre os níveis de representação (semântico e fonológico). Nos indivíduos com quadros mais amenos, manteve-se a integridade representacional e o fluxo de interação entre os níveis ativados, gerando, portanto, erros mistos, semânticos e fonológicos.
Dell et al. (1997, p.801) adotam a tese da continuidade (continuity hypothesis), que também é adotada na presente pesquisa. Segundo essa hipótese, a produção atípica seria uma extensão da variação natural encontrada em indivíduos sem lesão, havendo apenas graus diferentes de comprometimento no contínuo falante típico - atípico. Esse contínuo se aplica tanto se comparando afásicos e não afásicos quanto entre os afásicos.

Cuetos (2003) descreve a arquitetura funcional do sistema de produção oral através de um modelo com três níveis que se interrelacionam: (1) nível semântico ou de ativação do significado, onde conceitos estariam representados e categorizados dentro de um conjunto de características; (2) nível lexical ou de seleção e recuperação das palavras, onde se localizariam as representações das palavras e (3) nível fonológico, com as representações dos fonemas da língua e informações articulatórias necessárias para sua ativação junto aos órgãos fonoarticulatórios. 0 modelo compartilha os mesmos níveis e etapas de outros modelos anteriores como o de Levelt et al. (1999), por exemplo.

Portanto, há três processos importantes para nomeação. São eles: acesso semântico na ativação do conceito; processamento lexical, para acesso à palavra correspondente ao conceito ou significado; e processamento fonológico, para acesso à forma sonora da palavra (CUETOS, 2003). Uma alteração em qualquer dos processos gera dificuldades de nomeação, mas por causas distintas. Esses estágios de ativação de representações são feitos sucessivamente e essas relações podem explicar uma produção equivocada. Essas relações, envolvendo várias etapas, explicam algumas produções "trocadas" principalmente relacionadas a substituições semânticas: gato por cachorro ou calça por bermuda, ou mesmo as sonoras como tigre por trigo.

Cuetos (2009) e Ortiz (2010) diferenciam a etapa de reconhecimento da etapa de acesso lexical, da mesma maneira proposta em Pisoni et al. (1985). 
Para os autores, o reconhecimento seria uma primeira identificação em função de características acústicas e segmentais do item lexical e o acesso lexical envolveria a ativação de significados presentes no léxico do ouvinte. O significado é acessado no léxico após a identificação da forma sonora das representações no léxico.

Há também o modelo em cascata que defende a ativação simultânea dos níveis, sem o retorno das ativações. Já que não haveria retorno, o nível dos fonemas não influenciaria na seleção lexical, a ativação é simultânea dos níveis, mas unidirecional sem retorno para confirmar ou excluir ativações (RAPP e GOLDRICK, 2000). O que aconteceria nesse modelo em cascata seria a maior ou menor ativação de cada nível, explicando assim os tipos de erros produzidos. Por exemplo, uma ativação semântica alta geraria erros semânticos, ao passo que, se a maior ativação ocorrer no nível fonológico, as substituições seriam do tipo fonológico ou neologismos, e se ocorre menor ativação em ambos os níveis, consequentemente serão obtidas "não respostas".

Ainda, o modelo de reconhecimento auditivo de palavras TRACE, defendido por McClelland e Elman (1986), também propõe ligações diretas entre os itens lexicais. Um aumento no nível de ativação de um candidato, então, automaticamente leva a uma diminuição do nível de ativação de outros vizinhos. 0 modelo não estabelece a atuação de uma inibição bottom-up, no caso de não correspondência fonológica e/ou semântica entre input e entrada lexical, e entende que a ativação dos candidatos diminui gradativamente até alcançar o alvo, o item vencedor em relação aos vizinhos (winner-takes-all), o item a ser produzido pelo falante.

O que se mostra interessante é que as falhas de acesso lexical não são exatamente aleatórias, uma vez que as substituições correspondem a unidades linguísticas similares aos itens lexicais alvos. A troca entre palavras, por exemplo, na maioria das vezes, mantém a mesma categoria gramatical (GORDON, 2002; FAY e CUTLER, 1977; GARRETT, 1975, 1980; MacKAY, 1982 apud DELL et al. 1997) e segmentos entre alvo e substituição. Com relação à substituição por semelhança sonora, há semelhança entre os sons produzidos, indicando uma espécie de seleção de características fonológicas. Normalmente, consoantes são substituídas por consoantes e vogais por vogais e os inícios e/ou rimas são preservados (GORDON, 2002).

Já o modelo de nomeação a partir de estímulos visuais inclui a etapa de análise visual. A complexidade visual da figura é uma variável que atuaria nessa análise, ou seja, se há menos ou mais traços para facilitar ou dificultar o reconhecimento da imagem (SANFELIU e FERNANDEZ, 1996). Considera-se que o reconhecimento ocorre quando a figura é reconhecida como familiar dentre as representações que já se tem e que vão sendo acumuladas ao longo da vida.

Na nomeação de figuras, a atividade cerebral se inicia na área estriada do córtex occipital no lobo occipital (pelos processos de análise visual), e direciona-se ao lobo parietal, onde ocorre o reconhecimento. Ao identificar a representação, ativa-se o significado e, na área de Wernicke (no lobo temporal esquerdo), através do fascículo arqueado, ativa-se a área de Broca (no lobo frontal esquerdo), onde se elabora o programa articulatório para a produção da fala (Fig. 2). Identificar as áreas onde ocorrem essas etapas não é suficiente para compreender a complexidade do processamento linguístico (CUETOS, 2003). No caso de uma lesão cerebral ocorrida na região responsável pela linguagem, atingindo um dos lobos no hemisfério esquerdo, conforme na Figura 2, o processamento linguístico é alterado. Assim, o objetivo do terapeuta é, em um primeiro momento, considerando o sistema linguístico de pessoas sem lesão, estabelecer o que foi alterado na lesão. 
Figura 2 - Cérebro com as delimitações dos lobos e áreas (download: <http://www. sistemanervoso.com/pagina.php?secao=2\&materia_id=464\&materiaver=1>)

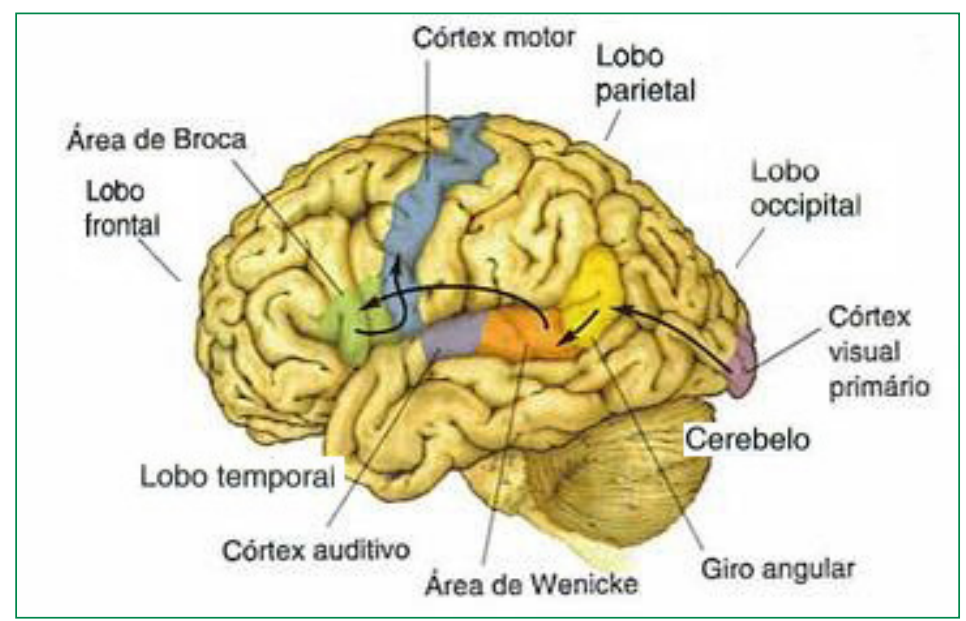

Fonte: SENNA (2013, p.33).

$\mathrm{Na}$ nomeação de figuras, diversos processos estão envolvidos: (a) análise visual, (b) reconhecimento da figura, (c) acesso ao significado, (d) recuperação do nome, (e) ativação dos segmentos sonoros e (f) articulação (CUETOS, 2003). A última etapa para emissão da fala, ou seja, a articulação, se decompõe em planejamento da sequência de movimentos, envio da informação ao cérebro para produção e atividade motora em si. Considerando o modelo conexionista, os conceitos são formados a partir de características perceptivas e motoras, e se encontram distribuídos em uma ampla zona cerebral correspondente, não havendo áreas específicas para a informação representada. Existem áreas convergentes na zona parietotemporal, com função específica perceptiva ou motora que são encarregadas de unir as características para formar o conceito; no entanto, são áreas flexíveis, modificáveis com o aprendizado (CUETOS, 2003).
Para Cuetos (2003), o uso dos itens lexicais influencia neurologicamente na sua recuperação. As conexões neuronais se fortaleceriam com o uso. Por outro lado, com o passar do tempo, e o inverso também aconteceria, ou seja, por uma diminuição da atividade geral do cérebro, a efetividade do acesso (eficácia das conexões) vai se perdendo. A frequência de ocorrência dos itens lexicais se constitui em um dos componentes que caracterizam o uso. Segundo BYBEE (1995), a frequência de ocorrência de um item lexical tem impacto na sua representação. Quanto mais frequente for um item, mais acessado será e sua representação será mais robusta. Há evidências de que itens mais frequentes tendem a resistir a mudanças analógicas que envolvem regularização de paradigmas morfológicos (BYBEE, 2015, p. 94-97). A hipótese é a de que a alta frequência de uso reforça as representações, preservando a forma da palavra na analogia. Diversos estudos têm demonstrado a importância do papel da frequência do item no acesso lexical (BARRY, MORRISON e ELLIS, 1997; MORRISON, ELLIS e QUINLAN, 1992; NOZARI et al., 2010). Segundo Levelt et al. (1999), o efeito de frequência das palavras, descoberto por Oldfield e Wingfield (1965), deve ser considerado na nomeação por influenciar determinados níveis de seu modelo.

De acordo com Cuetos (2003), na nomeação de figuras, também é importante considerar as variáveis das figuras que influenciam na nomeação como (a) complexidade visual da figura, (b) familiaridade com o item: relacionado ao contato diário, e influenciando mais no sistema semântico da produção oral na nomeação, gerando mais substituições semânticas, (c) imagética: facilidade ou dificuldade de imaginar o item, e influenciando também mais no sistema semântico, além da (d) frequência de uso, (e) comprimento do item (tamanho) e complexidade articulatória, (f) acordo de nome: referente ao consenso das pessoas em nomear uma figura, por exemplo: burro e asno, vagem e ervilha, etc., e (g) idade de aquisição: referente à ordem com que aprendemos as palavras ao longo 
da vida. Essa última variável é de difícil quantificação, mas mesmo assim deve ser considerada, já que tem sido verificada sua atuação na nomeação (BONIN, FAYOL e CHALARD, 2001; CUETOS, 2003). Segundo BONIN et al. (2001, p. 469-470), os itens lexicais adquiridos mais cedo são recuperados mais rapidamente da memória do que os adquiridos posteriormente. Ainda, segundo os autores, o efeito da idade de aquisição, observado tanto para produção oral quanto em tarefa de nomeação de figuras, é um achado bem estabelecido da Psicolinguística. Assim, a idade em que o item lexical foi adquirido pode ter influência quando se deseja nomear uma figura. Esse efeito pode ter relação com a proposição de Jakobson (1941), segundo a qual há relação entre a aquisição fonológica e perda na afasia. O desenvolvimento fonológico teria uma ordem universal e inata regulada por leis estruturais. As estruturas linguísticas adquiridas por último estariam mais sujeitas a substituições na produção controlada e estruturas adquiridas anteriormente tenderiam a ser mantidas.

As estimativas de frequência de palavras tendem a se correlacionar com as estimativas da idade de aquisição das palavras segundo Carroll e White (1973) e Morrison et al. (1992). Segundo Levelt et al. (1999), ambos os fatores influenciam e devem ser analisados. Em estudo de Cuetos, Aguado, Izura e Ellis (2002), com afásicos usando a tarefa de nomeação de desenhos, foi observada a influência da variável idade de aquisição, sendo esta mais influente que a frequência de uso e a familiaridade. Belke, Brysbaert, Meyer e Ghyselinck (2005) também identificaram a importância da variável idade de aquisição. Morrison et al. (1992), comparando nomeação de objetos e os tempos de categorização, argumentaram que o efeito da idade de aquisição influi no acesso às formas fonológicas.

A seção a seguir apresenta as questões de trabalho e a metodologia empregada na coleta e análise dos dados.

\section{Objetivos, hipóteses e metodologia}

A pesquisa parte da hipótese dos Modelos Baseados no Uso segundo a qual o léxico se organiza multidimensionalmente, em redes de conexões lexicais (NetworkModel) em função de similaridades sonoras e semânticas (cf. BYBEE, 1995, 2010). Além disso, assume-se que a experiência com o uso linguístico afeta a representação lexical. Assim, a maior ou menor acurácia na produção espontânea ou em testes de nomeação se relacionaria com as hipóteses de organização do léxico em função de alguns fatores que poderiam influenciar a organização lexical e consequentemente no acesso para a produção como, por exemplo, a frequência de ocorrência do item alvo. A frequência de uso captura um aspecto da experiência, uma vez que a frequência com que um item é usado tem impacto na robustez da sua representação (PIERREHUMBERT, 2003; 2016). Ainda, uma vez que a ativação de um item implica na ativação de outros que compartilham semelhança sonora e/ou semântica, e, portanto, participam da mesma rede ou esquema lexical, as substituições podem preservar informações como tamanho do item lexical (número de sílabas), segmentos ou categoria semântica, conforme já mencionado anteriormente.

A pesquisa teve por objetivo investigar o acesso lexical e representação em indivíduos afásicos. As variáveis de análise, variáveis dependentes, foram tipo de resposta e tempo de resposta. Neste artigo serão analisados somente os resultados relativos ao tipo de resposta. As variáveis independentes relacionadas com os itens alvo foram: tamanho do item, frequência de ocorrência do item, idade de aquisição, familiaridade e imageabilidade. Também foram verificadas variáveis relacionadas com os indivíduos como diagnóstico, tempo de terapia, tipo de lesão, entre outros. Neste artigo serão comentados os resultados obtidos para as variáveis linguísticas que se mostraram mais significativas no grupo dos afásicos do ponto de vista 
estatístico através da regressão logística²: idade de aquisição, familiaridade e frequência de ocorrência do item (SENNA, 2013, p. 87).

Os dados foram obtidos através de um Teste de Nomeação composto de 154 figuras selecionadas das 260 figuras originais elaborado com essa finalidade (SNODGRASS e VANDERWARTS, 1980), aplicado a 23 indivíduos afásicos e 23 controles $^{3}$. As figuras do teste original têm sido utilizadas por pesquisas em diversas línguas como o inglês (BARRY, MORRISON e ELLIS, 1997), o espanhol (CUETOS et al. 1999) e o francês (ALARIO e FERRAND 1999) e foram padronizadas para o português brasileiro em variáveis como acordo de nome, complexidade visual e familiaridade por Pompéia, Miranda e Bueno (2001). No entanto, para a realização da presente pesquisa também foi aplicado um teste de acordo de nome para 36 falantes do português brasileiro a fim de dar maior confiabilidade às figuras selecionadas, auxiliando na escolha das figuras alvo, que resultou na seleção das 154 das 260 originais para a tarefa de nomeação. Também foi aplicado um teste para avaliação de idade de aquisição dos itens selecionados no acordo de nome.

Com relação à avaliação do acordo de nome, os voluntários, de nível universitário e com média de 31 anos de idade, foram solicitados a nomear as 260 figuras de Snodgrass e Vanderwarts (1980). Os resultados obtidos indicaram que 98 figuras foram nomeadas da mesma maneira por todos os voluntários (100\% de acordo de nome), 26 figuras por apenas 31 dos voluntários (97\% de acordo de nome), 24 figuras por 30 indivíduos (94\% de acordo de nome) e 14 figuras por 29 indivíduos (91\% de acordo de nome), totalizando 161 figuras representando itens alvo com mais de $90 \%$

${ }^{2}$ A técnica do Modelo de Regressão Logística para variáveis dependentes visa uma predição de valores (variáveis categóricas) a partir de variáveis contínuas ou binárias ( 0 ou 1), possibilitando colocar variáveis em concorrência e eleger assim a variável que melhor explica ou informa acerca da relação com outra, um modelo. O objetivo do Modelo de Regressão Logística é estimar a probabilidade de uma determinada categoria, no caso correspondente a variável dependente que é dicotômica (no valor 1). Federal do Rio de Janeiro, no de registro 009/09, em 2009. de acordo de nome dentre as 260 figuras originais. As figuras relacionadas a substantivos compostos como "carrinho de bebê", "chave de fenda", "lata de lixo", "tábua de passar", entre outros, também foram retiradas, resultando em 154 dos 260 itens originais para o Teste de Nomeação.

O estabelecimento da idade de aquisição foi realizado a partir das 154 figuras selecionadas utilizando a metodologia aplicada por Cuetos et al. (2010). Os valores de idade de aquisição são estimativas de quando os itens alvo foram adquiridos. Esses valores foram estabelecidos através de um questionário submetido a 128 voluntários, indivíduos sem lesão e, em sua maioria, de nível universitário. Os voluntários foram divididos em dois grupos. 0 Grupo 1 foi composto por 60 indivíduos que responderam à estimativa por intervalos de idade (antes dos 2 anos, entre 2 e 4 anos, entre 4 e 6 anos, entre 6 e 8 anos, entre 8 e 10, entre 10 e 12 ou mais de 13 anos). Pela dificuldade em preencher quando foi exatamente a aquisição, a orientação dada pelo examinador foi a de não pensar muito e sim escolher como intuição. 0 Grupo 2 foi composto por 68 indivíduos que responderam utilizando uma escala de 1 a 7 , que não especifica exatamente a idade de aquisição, mas estabelece uma ideia de gradualidade: 1 corresponde a um item adquirido muito cedo e 7, mais tarde. Um estudo estatístico comparativo (Correspondências, Fatorial e de Componentes Principais) permitiu verificar a concordância entre as duas medidas, pelo menos no que diz respeito ao que deve ser tomado como uma medida da primitividade de idade de aquisição. Para uma descrição mais detalhada desta metodologia, ver Camiz et al. (2015).

Os voluntários eram pacientes particulares e do ambulatório de afasia do curso de Fonoaudiologia da Universidade Federal do Rio de Janeiro entre 2011 e 2012, sendo 12 mulheres e 11 homens, e 23 indivíduos do grupo controle, sendo 12 mulheres e 11 homens, falantes do português brasileiro, escolhidos considerando alguns critérios como faixa etária, sexo 
e escolaridade, para manter a homogeneidade em relação aos afásicos. Além disso, históricos neurológicos ou psiquiátricos foram considerados como fatores impeditivos de participação como controle na pesquisa. O ANEXo I e o ANEXo II apresentam, respectivamente, as informações sobre os dois grupos relativas à idade, sexo e escolaridade dos indivíduos que compõem a amostra, bem como as informações específicas do grupo dos afásicos, relativas ao diagnóstico, tempo de lesão, e desempenho nos testes de avaliação de compreensão oral e de produção oral e de rastreamento cognitivo.

Para a avaliação da compreensão oral dos afásicos, foi escolhida a tarefa de associação fala-desenho do teste Montreal Toulouse versão alpha, adaptado por Leonor Scliar-Cabral (1981), em que o paciente, ao escutar o avaliador, deve sinalizar, entre as 4 ou 6 opções desenhadas (dependendo do estímulo), aquela que representa o que ele ouviu e compreendeu. A avaliação é constituída de 5 palavras, 3 orações simples e 3 complexas e possui distratores semânticos, fonológicos e visuais. Para avaliação da produção, também foi utilizada a bateria de repetição do teste Montreal Toulouse versão alpha. A testagem consistiu na repetição de 8 palavras e 3 frases $(1$ frase simples e 2 mais complexas e mais longas). A pontuação do Mini Exame do Estado Mental (Mini-mental), um exame neuropsicológico de rastreamento cognitivo (BRUCKI et al. 2003), também foi coletada do acervo fonoterápico de cada paciente. $\mathrm{O}$ exame consta de avaliação da orientação temporal e espacial, memória imediata, atenção e cálculo, evocação e linguagem. Como resultado do Mini-mental se obtém uma pontuação que diante da esperada (segundo a escolaridade) detecta ou não algum comprometimento das habilidades cognitivas testadas, possibilitando melhor caracterização dos participantes ou até mesmo a exclusão de sua participação. Diante da pontuação obtida nesses três testes de habilidades importantes os voluntários afásicos foram caracterizados. Os escores obtidos para os indivíduos nos testes também foram utilizados como variáveis independentes para análise de desempenho na tarefa de nomeação (cf. Senna, 2013, p. 112-113). Conforme mencionado anteriormente, somente serão abordados neste artigo os resultados relativos a três variáveis relacionadas ao item alvo.

A coleta de dados foi realizada no ambiente terapêutico do paciente. Para aplicação do teste, foi utilizado o software DMDX, disponível gratuitamente em <http://www.u.arizona.edu/ kforster/dmdx/dmdx.htm>. O software permite controle experimental e tempo de exposição do estímulo, sendo possível mensurar os tempos de reação aos estímulos visuais ou auditivos. Foi necessário o controle diferenciado de tempo de exibição de cada figura na tela para nomeação para que o experimento não se tornasse cansativo para o grupo de voluntários, nem frustrasse os afásicos diante de alguma dificuldade. Cada figura ficou exposta cerca de 8 segundos para os afásicos e aproximadamente 4 segundos para os voluntários sem lesão. Cada voluntário recebeu um fone de ouvido com microfone e, diante do notebook HP G42212BR, era orientado verbalmente a realizar o experimento, nomeando as figuras conforme se apresentavam. Após as instruções duas figuras que não fazem parte do teste foram apresentadas como fase de treino. Imediatamente após o treino, as 154 figuras selecionadas foram apresentadas aleatoriamente.

Na análise, utilizou-se o checkvocal, um aplicativo do Windows (PROTOPAPAS, 2007), que facilita a verificação da acurácia, possibilitando a quantificação dos tempos de resposta e a gravação das produções em tarefas experimentais. A transcrição para posterior análise quantitativa e qualitativa dos dados foi realizada pela própria pesquisadora em dois momentos para conferência e eliminação de possíveis ambiguidades. $\mathrm{O}$ formato do arquivo de gravação de áudio, para posterior análise, foi ".wav".

Os valores de frequência de ocorrência dos itens relacionados às figuras do Teste de Nomeação foram obtidos e controlados utilizando o corpus NILC/ São Carlos - Projecto AC/DC com 32.3 milhões de palavras (<http://www. linguateca.pt/acesso/corpus.php? corpus=SAOCARLOS $>$ ), que mensura a 
frequência do item por classe gramatical. Assim, o item "cerca" é contabilizado diferentemente como substantivo ou como parte da locução "cerca de". Os valores de complexidade visual e familiaridade utilizados na presente pesquisa são os obtidos na padronização de variáveis para o português de Pompéia, Miranda e Bueno (2001) que incluía também as outras figuras de Snodgrass e Vanderwart (1980). Os estudos (SNODGRASS e VANDERWART, 1980; POMPÉIA, MIRANDA e BUENO, 2001) sugerem relação inversa entre essas duas variáveis. Os sujeitos atribuem menos complexidade às figuras mais familiares.

As respostas foram classificadas como corretas, no caso de a produção ser igual ao item alvo da figura e, caso contrário, foram definidas em função do tipo de substituição como parafasia semântica, fonológica ou mista, sem relação com o alvo, pseudopalavras, ininteligível, vazio (omissões), termo genérico, circunlocução ou outro alvo. O software livre R-Project (www.rproject.org) foi empregado no tratamento estatístico dos dados para verificar a dependência entre variáveis independentes e variável dependente (teste de qui-quadrado $)^{4}$ e a significância estatística das variáveis independentes (regressão logística).

\section{Análise dos resultados}

Foram obtidos 3.542 dados no grupo dos afásicos e 3.543 no grupo controle. Com relação aos tipos de respostas, os dois grupos se caracterizam por uma predominância de respostas corretas em comparação com os demais tipos de substituição, conforme pode ser observado na Tabela 1 a seguir.

4 O teste de qui-quadrado testa a hipótese de independência de duas variáveis qualitativas. Se a hipótese nula for verdadeira, há independência entre as variáveis, ou seja, não relação entre a ocorrência de uma em relação à outra. 0 p-valor indica se há ou não dependência entre as variáveis. Há dependência entre as variáveis, rejeição da hipótese nula, quando o p-valor é menor que o nível de significância adotado,
Tabela 1 - Distribuição dos tipos de respostas ao Teste de Nomeação em afásicos e controles

\begin{tabular}{lccccc}
\hline & $\begin{array}{c}\text { Tipos de } \\
\text { resposta }\end{array}$ & Corretas & Semânticas & Fonológicas & $\begin{array}{c}\text { Outras } \\
\text { respostas }\end{array}$ \\
Voluntários & $54,4 \%$ & $8,0 \%$ & $9,8 \%$ & $27,8 \%$ \\
Afásicos & $94,4 \%$ & $2,2 \%$ & $0,5 \%$ & $2,9 \%$ \\
\hline Controles & & & & \\
\hline
\end{tabular}

X-squared $=42.644 ; \mathrm{df}=3 ; \mathrm{p}$-value $=2.929 \mathrm{e}-09$.

Os percentuais obtidos em cada categoria apontam para uma diferença quantitativa entre os dois tipos de voluntários e o tipo de resposta. $0 \mathrm{p}$-valor= 2.929e-09 indica que há relação entre tipo de voluntário e tipo de resposta. As substituições sem relação com o alvo, pseudopalavras, ininteligível, vazio (omissões), termo genérico, circunlocução ou outro alvo foram reagrupadas em uma única categoria denominada "Outras respostas". Do exposto na tabela, observam-se os mesmos tipos de substituições nos dois grupos, isto é, as substituições produzidas por afásicos são também realizadas pelo grupo controle. 0 que difere os grupos é a distribuição de respostas de acordo com o alvo e substituições. Há, portanto, uma diferença quantitativa, e não qualitativa, entre os dois grupos. Esse resultado corrobora a tese de continuidade de Dell et al. (1997), mencionada anteriormente, segundo a qual a diferença nas falhas de acesso lexical entre indivíduos com lesão e sem lesão reside em diferença quantitativa e não quanto à natureza das substituições.

O teste de regressão logística (SENNA, 2013, p. 87) selecionou como variáveis mais influentes, nos tipos de respostas da tarefa de nomeação de figuras dos afásicos, a idade de aquisição do item ( $p$-valor $=<2 \mathrm{e}-16)$, a familiaridade ( $p$-valor=4.51e-06) e a frequência do item alvo ( $p$-valor= 0.000776). Observa-se, em função dos p-valores obtidos, que a idade de aquisição se mostrou uma variável mais influente que a frequência de 
ocorrência do item na recuperação lexical, ratificando a ideia de "espelho invertido" de Jakobson (1941 apud SCARPA, 2005), segundo a qual as estruturas linguísticas adquiridas primeiro são as que mais se preservam em situações de perda linguística. A relevância estatística da variável frequência do item lexical no grupo dos indivíduos com afasia ratifica também a hipótese adotada na pesquisa de que o desempenho em testes de nomeação se relaciona com as hipóteses de organização do léxico em representações baseadas na experiência do indivíduo com o uso linguístico.

A Tabela 2 a seguir apresenta os resultados obtidos para o tipo de resposta em função da idade de aquisição dos itens lexicais.

Tabela 2 - Tipos de resposta por Idade de Aquisição do item alvo na tarefa de nomeação dos afásicos

\begin{tabular}{lccccccccccc}
\hline & $\begin{array}{c}\text { Tipos de } \\
\text { resposta }\end{array}$ & \multicolumn{2}{c}{ Corretas } & \multicolumn{2}{c}{ Semânticas } & Fonológicas & \multicolumn{2}{c}{$\begin{array}{c}\text { Outras } \\
\text { respostas }\end{array}$} & \multicolumn{2}{c}{ Total } \\
\cline { 2 - 13 } $\begin{array}{l}\text { Idade de } \\
\text { aquisição }\end{array}$ & $\mathbf{N}$ & $\mathbf{\%}$ & $\mathbf{N}$ & $\mathbf{\%}$ & $\mathbf{N}$ & $\mathbf{\%}$ & $\mathbf{N}$ & $\mathbf{\%}$ & $\mathbf{N}$ & $\%$ \\
Muito Cedo & 490 & 68,7 & 58 & 8,1 & 41 & 5,8 & 124 & 17,4 & 713 & 100 \\
Cedo & 828 & 59 & 94 & 6,7 & 119 & 8,5 & 362 & 25,8 & 1403 & 100 \\
Tardia & 322 & 48,3 & 58 & 8,7 & 84 & 12,6 & 203 & 30,4 & 667 & 100 \\
Muito Tarde & 251 & 33,1 & 73 & 9,6 & 102 & 13,4 & 333 & 43,9 & 759 & 100 \\
Total & 1891 & 53,4 & 283 & 8,0 & 346 & 9,8 & 1022 & 28,9 & 3542 & \\
\hline
\end{tabular}

Qui-quadrado = 234.559; grau de liberdade $=9 ;$ - valor $<2.2 \mathrm{e}-16$.

A variável idade de aquisição do item lexical permite avaliar o desempenho no teste em função da probabilidade de acerto (sucesso) ou erro (falha) no acesso lexical. Os valores obtidos no questionário de avaliação da idade de aquisição foram organizados em quartis, o que possibilitou o enquadramento dos itens em 4 categorias de idade de aquisição: muito cedo, cedo, tardia, muito tarde. 0 p-valor obtido é indicativo da significância estatística desta variável, isto é, indica que há relação entre idade de aquisição e tipo de resposta. A porcentagem de respostas corretas diminui quanto mais tardia for a idade de aquisição dos itens, de 68,7 a 33,1\%, e aumenta a porcentagem de substituições fonológicas, de 17,4 a 43,9\%, e de outras respostas, em que não há acesso a nenhuma informação do item alvo. As substituições semânticas se mantêm em torno de um mesmo percentual, entre 6,7\% e 9,6\%. Esses resultados estão na direção da hipótese de Jakobson (1941), segundo a qual o conhecimento linguístico adquirido primeiro é o que mais se preserva em situação de perdas linguísticas.

A Tabela 3 a seguir apresenta os tipos de resposta por familiaridade dos itens de acordo com valores padronizados por Pompéia, Miranda e Bueno (2001). Os escores de familiaridade foram agrupados em duas categorias: menos familiar e mais familiar.

Tabela 3 - Tipos de resposta por Familiaridade dos itens na tarefa de nomeação dos afásicos

\begin{tabular}{lccccccccccc}
\hline $\begin{array}{r}\text { Tipos de } \\
\text { resposta }\end{array}$ & Corretas & \multicolumn{2}{c}{ Semânticas } & Fonológicas & \multicolumn{2}{c}{$\begin{array}{c}\text { Outras } \\
\text { respostas }\end{array}$} & \multicolumn{2}{c}{ Total } \\
$\begin{array}{l}\text { Grau de } \\
\text { familiaridade }\end{array}$ & $\mathbf{N}$ & $\mathbf{\%}$ & $\mathbf{N}$ & $\mathbf{\%}$ & $\mathbf{N}$ & $\mathbf{\%}$ & $\mathbf{N}$ & $\mathbf{\%}$ & $\mathbf{N}$ & $\mathbf{\%}$ \\
Menos familiar & 625 & 43,1 & 121 & 8,4 & 162 & 11,2 & 541 & 37,3 & 1449 & 100 \\
Mais familiar & 1266 & 60,5 & 162 & 7,7 & 184 & 8,8 & 481 & 23 & 2093 & 100 \\
Total & 1891 & 53,4 & 283 & 8,0 & 346 & 9,8 & 1022 & 28,9 & 3542 & \\
\hline
\end{tabular}

Qui-quadrado $=114.8494$, grau de liberdade $=3$ e p-valor $<2.2 \mathrm{e}-16$.

De acordo com o p-valor obtido, observa-se que há relação entre grau de familiaridade com o item e tipo de resposta no Teste de Nomeação dos afásicos. $\mathrm{O}$ p-valor baixo indica que o acesso lexical foi influenciado por esta variável nos indivíduos afásicos. Quanto mais familiar o item alvo, maior a porcentagens de respostas corretas, de 43,1 a 60,5\%. Também se observa 
que as palavras menos familiares apresentaram maior percentual de outras respostas, 37,3\%, não relacionadas semântica ou fonologicamente com o item alvo, que as palavras mais familiares, $23 \%$. As diferenças percentuais das substituições semânticas e fonológicas não são muito acentuadas em função do grau de familiaridade, no entanto, os percentuais mais altos dos dois tipos de substituição ocorrem com as palavras menos familiares. Esses resultados corroboram os encontrados em Cuetos et al. (2002) em relação à variável familiaridade.

Conforme mencionado anteriormente, também foi verificada a relação entre tipo de resposta e frequência de ocorrência dos itens alvo. A Tabela 4 a seguir apresenta a distribuição das respostas em função desta variável. Os itens alvo de alto acordo de nome foram classificados em quatro grandes grupos de frequência: muito frequente, frequente, pouco frequente e muito pouco frequente, obtidos através da distribuição dos valores em quartis.

Tabela 4 - Tipos de resposta por Frequência dos itens na tarefa de nomeação dos afásicos.

\begin{tabular}{lccccccccccc}
\hline $\begin{array}{c}\text { Tipos de } \\
\text { resposta }\end{array}$ & Corretas & Semânticas & Fonológicas & $\begin{array}{c}\text { Outras } \\
\text { respostas }\end{array}$ & \multicolumn{2}{c}{ Total } \\
\cline { 2 - 12 } $\begin{array}{l}\text { Frequência } \\
\text { de ocorrência }\end{array}$ & $\mathbf{N}$ & $\mathbf{\%}$ & $\mathbf{N}$ & $\mathbf{\%}$ & $\mathbf{N}$ & $\mathbf{\%}$ & $\mathbf{N}$ & $\mathbf{\%}$ & $\mathbf{N}$ & $\%$ \\
\hline Muito Pouco & 303 & 38,7 & 60 & 7,7 & 109 & 13,9 & 310 & 39,6 & 782 & 99,9 \\
Pouco Frequente & 624 & 50,2 & 115 & 9,3 & 124 & 10 & 379 & 30,5 & 1242 & 100 \\
Frequente & 611 & 61,8 & 67 & 6,8 & 81 & 8,2 & 230 & 23,3 & 989 & 100 \\
Muito Frequente & 353 & 66,7 & 41 & 7,8 & 32 & 6 & 103 & 19,5 & 529 & 100 \\
Total & 1891 & 53,4 & 283 & 8,0 & 346 & 9,8 & 1022 & 28,9 & 3542 & \\
\hline
\end{tabular}

Qui-quadrado = 152.4618; grau de liberdade $=9 ; p$-valor $=<2.2 \mathrm{e}-16$.

Há relação entre a frequência de ocorrência dos itens alvo e os tipos de resposta no Teste de Nomeação de figuras dos afásicos, confirmado pelo p-valor obtido no teste de qui-quadrado. Quanto mais frequente o item lexical, maior porcentagem de respostas corretas, de $38,7 \%$ a $66,7 \%$. 0 acesso lexical é facilitado quando o item é muito frequente, por isso aumenta a porcentagem de acertos. Esses resultados replicam os de outros estudos que também encontram a influência da frequência na nomeação, como Barry, Morrison e Ellis (1997), Cuetos et al. (2002);Belke et al. (2005), Nozari et al. (2010), entre outros. Também se observa que predominam as substituições do tipo "outras respostas" em todos os grupos de frequência. As substituições fonológicas ocorrem mais frequentemente nos itens alvo de baixa frequência. Já as respostas em que há acesso à informação semântica se mantêm com percentual equivalente tanto em itens muito frequentes quanto muito pouco frequentes. Os resultados apontam para influência do efeito de frequência de ocorrência no acesso lexical, uma vez que o desempenho no Teste de Nomeação de figuras melhora nos itens caracterizados como Frequente e Mais Frequente.

0 acesso lexical envolve tanto acesso à informação semântica quanto à forma sonora do item lexical. Quanto ao efeito das variáveis estudadas em relação ao tipo de substituição, a literatura tem mostrado que efeitos de frequência dos itens (SCHWARTZ et al., 2004) quanto de idade de aquisição (BARRY et al., 2001) tendem a se manifestar em relação à busca da forma fonológica. Os resultados obtidos na pesquisa mostraram que, de fato, as substituições semânticas não diferem em função da frequência do item e que somente nos itens de mais baixa frequência houve mais substituições fonológicas $(13,9 \%)$, havendo um decréscimo em função do aumento da frequência do item. No entanto, Kittredge et al. (2008) apresentam evidências de desempenho, em teste de nomeação de indivíduos afásicos e controles, que apontam para efeito de frequência também em substituições semânticas. Já a variável idade de aquisição é referida na literatura como afetando a representação e a organização da rede de relações lexicais desde 
seu início (ELLIS e LAMBON RALPH, 2000). O resultado observado aponta a ausência de relação entre substituição semântica e idade de aquisição do item, uma vez que os percentuais são muito próximos nas quatro categorias apresentadas na Tabela 2, replicando os achados de Kittredge et al. (2008).

\section{Considerações finais}

As evidências encontradas nos diversos estudos utilizando tarefa nomeação com indivíduos afásicos, aliadas às hipóteses de organização lexical em redes, baseadas em similaridades semânticas e sonoras das palavras, dos Modelos baseados no Uso, permitem concluir que as substituições de afásicos não são aleatórias, ao mesmo tempo em que ratificam a proposta de organização lexical em redes. As falhas de acesso lexical dos voluntários afásicos da pesquisa no Teste de Nomeação de Figuras geraram algumas substituições semelhantes a um "vizinho" do item alvo em relação à forma sonora (substituições fonológicas). Com isso, o fator densidade de vizinhança, não abordado no presente estudo, também deve ser considerado nos estudos sobre acesso lexical em afásicos (Gordon, 2002). Vitevitch e Stamer (2006) definem densidade de vizinhança como referente ao número de representações lexicais que compartilham determinadas semelhanças com a palavra alvo. A maior ou menor densidade de vizinhança lexical corresponde à quantidade de itens que compartilham algum tipo de semelhança sonora ou semântica. Um item com muitos vizinhos teria uma maior concorrência no acesso lexical.

Os resultados aqui apresentados também acrescentam evidências à hipótese da continuidade entre população típica e atípica no acesso lexical, uma vez que houve diferença quantitativa e não qualitativa entre os dois grupos estudados em relação à distribuição dos tipos de resposta. Os resultados apontaram como variáveis mais influentes na tarefa de nomeação pelos afásicos a idade de aquisição, a familiaridade e a frequência do item alvo. Este estudo buscou contribuir para o entendimento do acesso lexical na Afasia, além de ser o primeiro a abordar a questão metodológica do estabelecimento da idade de aquisição dos itens lexicais do português brasileiro.

\section{Referências}

ALARIO, François-Xavier; FERRAND, Ludovic. A set of pictures standardized for French: Norms for name agreement, image agreement, familiarity, visual complexity, image variability, and age of acquisition. Behavior Research Methods, Instruments, \& Computers, v. 31, p. 531-552, 1999. https://doi.org/10.3758/BF03200732

ALVES, Daniel. P. Pistas prosódicas no acesso on-line de falantes adultos do português brasileiro. 2010. 139 fl. Dissertação (Mestrado em Linguística) - Universidade Federal de Juiz de Fora, Juiz de Fora, 2010.

BARRY, Christopher; MORRISON Catriona M.; ELLIS, Andrew W. Naming the Snodgrass and Vanderwart Pictures: Effects of Age of Acquisition, Frequency, and Name Agreement. The Quarterly Journal of Experimental Psychology, v. 50, n 3, p. 560-585, 1997. https:// doi.org/10.1080/783663595

BARRY, Christopher; HIRSH, Katherine W.; JOHNSTON, Robert A.; WILLIAMS, Catherine L. Age of acquisition, word frequency, and the locus of repetition priming of picture naming. Journal of Memory and Language, v. 44, p. 350-375, 2001. https://doi.org/10.1006/ jmla.2000.2743

BELKE, Eva; BRYSBAERT, Marc; MEYER, Antje; GHYSELINCK, Mandy. Age of acquisition effects in picture naming: evidence for a lexical-semantic competition hypothesis. Cognition, v. 96, n 2, p. 45-54, 2005. https://doi.org/10.1016/j.cognition.2004.11.006

BONIN, Patrick; FAYOL, Michel; CHALARD, Marylène. Age of acquisition and word frequency in written picture naming. The Quarterly Journal of Experimental Psychology, v. 54, n 2, p. 469-489, 2001. https://doi.org/10.1080/713755968

BRUCKI, Sonia; NITRINI, Ricardo; CARAMELLI, Paulo; BERTOLUCCI, Paulo; OKAMOTO, Ivan. Sugestões para o uso do mini-exame do estado mental no Brasil. Arquivos de Neuropsiquiatria, v. 61, n. 3B, p. 777-781, set 2003. https://doi.org/10.1590/S0004282X2003000500014 
BYBEE, Joan. Regular Morphology and the Lexicon. Language and Cognitive Processes, v. 10, n. 5, p. 425-455, 1995. https://doi.org/10.1080/01690969508407111

BYBEE, Joan. Language, usage and cognition. Cambridge: Cambridge University Press, 2010.

BYBEE, Joan. Language Change. Cambridge: Cambridge University Press, 2015.

CAMIZ, Sergio; GOMES, Gastão C.; SENNA Fernanda D.; GOMES, Christina A. Using exploratory analysis to select judges and create components of a naming test to study aphasia. Discrete Applied Mathematics, v. 197, p. 114-122, 2015. https://doi.org/10.1016/j. dam.2015.05.018

CAPLAN, David. Neurolinguistics and linguistic aphasiology. Cambridge University Press: Cambridge, 1987.

CARROLL, John B.; WHITE, Margareth N. Age-of-acquisition norms for 220 picturable nouns. Journal of Verbal Learning and Verbal Behavior, v. 12, n 5, p. 563-576, 1973. https://doi.org/10.1016/S0022-5371(73)80036-2

COUDRY, Maria Irma H. Diário de Narciso - Discurso e Afasia. São Paulo: Martins Fontes, 1988.

CUETOS, Fernando V. ANOMIA - La dificultad para recorder las palavras. Madrid: Tea Ediciones, 2003.

CUETOS, Fernando V.; AGUADO, Gerardo; IZURA, Cristina; ELLIS, Andrew W. Aphasic naming in Spanish: predictors and errors. Brain and Language, v. 82, p. 344-365, 2002. https://doi.org/10.1016/S0093-934X(02)00038-X

CUETOS, Fernando; ELLIS, Andrew W.; ÁLVAREZ, Bernardo. Naming times for the Snodgrass and Vanderwart pictures in Spanish. Behavior Research Methods, Instruments, \& Computers, v. 31, p. 650-658, 1999. https://doi.org/10.3758/BF03200741

CUETOS, Fernando V., NOSTI, María G.; JIMÉNEZ, Luis; MANTIÑÁN, Nancy; OLMEDO, Alicia; CHOCANO, Alejandro D. ¿Síndromes o síntomas en la evaluación de los Afásicos? Psicothema. v. 22, n. 4, p. 715-719.

DELL, Gary S.; SCHWARTZ, Myrna F.; MARTIN, Nadine; SAFRAN, Eleonor F.; GAGNON, Deborah A. Lexical access in aphasic and non-aphasic speakers. Psychological Review, v. 104, n. 4 , p. $801-838,1997$.

DRUMMOND, Claudia. Reabilitação dos problemas de produção verbal nas afasias: do geral ao fonoaudiológico. In: CAPOVILLA, Fernando; MACEDO, Elizeu C. (Org.). Temas em Neuropsicolinguística, São Paulo: Tecmedd, p. 181-196, 2016.
ELLIS, Andrew; LAMBON RALPH, Mathews. Age of acquisition effects in adult lexical processing reflects loss of plasticity in maturing systems: Insights from connectionist networks. Journal of Experimental Psychology: Learning, Memory, and Cognition, v. 26, p. 1103-1123, 2000. https://doi.org/10.1037/0278-7393.26.5.1103

FEIDEN, Juliana. A. O acesso lexical na afasia: anomia, parafasia e estratégias comunicativas na produção oral. 2014. 147 fl. Dissertação (Mestrado em Letras) -Universidade Federal do Rio Grande do Sul, Porto Alegre, 2014.

FRANÇA, Aniela I.; LEMLE, Miriam; GESUALDI, Aline da R.; CAGY, Mauricio; INFANTOSI, Antonio Fernando C. A neurofisiologia do acesso lexical: palavras em português. Revista Veredas, v. 12, n. 2, p. 34-49, 2008.

GANDOLFO, Monica. C. A classificação das afasias em questão: lugares de institucionalização e de questionamento. 2006. 180 fl. Tese (Doutorado em Linguística) Universidade Estadual de Campinas, Campinas, 2006.

GOODGLASS, Harold; WINGFIELD, Arthur; HYDE, Mary R.; GLEASON, Jean B.; BOWLES, Nancy L.; GALLAGHER, Roberta E. The importance of word-initial phonology: Error patterns in prolonged naming efforts by aphasic patients. Journal of the International Neuropsychological Society, v. 3, n 2, p. 128-138, 1997.

GORDON, Jean K. Phonological neighborhood effects in aphasic speech errors: spontaneous and structured contexts. Brain and Language, v. 82, p. 113-145, 2002. https://doi.org/10.1016/S0093-934X(02)00001-9

KITTREDGE, Audrey; DELL, Gary S.; VERKULLEN, Jay; SCHWARTZ, Myrna. Where is the effect of frequency in word production? Insights from aphasic picture naming errors. Cognitive Neuropsychology, v. 25, n. 4, p. 463-492, 2008. https://doi. org/10.1080/02643290701674851

LEVELT, Willem. J. M.; ROELOFS, Ardy; MEYER, Antje S. A theory of lexical access in speech production. Brain and Behavioral Science, v. 22, p. 1-75, 1999.

LIMA, Bruna. S. Afasia e Linguagem figurada: o acesso lexical dentro de contextos metafóricos. 2010. $303 \mathrm{fl}$. Dissertação (Mestrado em Semiótica e Linguística) Universidade de São Paulo, São Paulo, 2010

MANSUR, Letícia L.; MACHADO, Thaís. H. Afasias: Visão Multidimensional da Atuação do Fonoaudiólogo. In: FERREIRA, Leslie P.; BEFI-LOPES, Débora Maria; LIMONGI, Suely Cecília O. (Org.). Tratado de fonoaudiologia, São Paulo: Roca, 2004. p. 392-401.

MCCLELLAND, James L.; ELMAN, Jeffrey L. The TRACE model of speech perception. Cognitive Psychology, v. 18, p. 1-86, 1986. https://doi.org/10.1016/0010-0285(86)90015-0 
MEDEIROS, Joyse; WEISSHEIMER, Janaina; FRANÇA, Aniela I.; RIBEIRO, Sidarta. Acesso lexical: uma rota dupla para o português brasileiro. Fórum Linguístico, v. 11, n 3, p. 278-292, jul./set. 2014. https://doi.org/10.5007/1984-8412.2014v11n3p278

MORRISON Catriona M.; ELLIS Andrew W.; QUINLAN Philip T. Age of acquisition, not word frequency, affects object naming, not object recognition. Memory and Cognition, v. 20, n. 6, p. 705-714, 1992. https://doi.org/10.3758/BF03202720

NOZARI, Nazbanou; KITTREDGE, Audrey K.; DELL, Gary S.; SCHWARTZ, Myrna. F. Naming and repetition in aphasia: Steps, routes, and frequency effects. Journal of Memory and Language, v. 63, n 4, p. 541-559, 2010. https://doi.org/10.1016/j.jml.2010.08.001

OLDFIELD, Richard. C.; WINGFIELD, Arthur. Response latencies in naming objects. Quarterly Journal of Experimental Psychology, v. 17, p. 273-281.

ORTIZ, Karin Z. Avaliação das Afasias. In: ORTIZ, Karin Z. Distúrbios Neurológicos Adquiridos: Linguagem e Cognição. São Paulo: Ed. Manole, p. 65-93, 2010.

PISONI, David. B.; NUSBAUM Hoeard.C.; LUCE Paul A.; SLOWIACZEK Louise M. Speech Perception, word recognition and the structure of the lexicon. Speech Communication, v. 4, p. 75-95, 1985. https://doi.org/10.1016/0167-6393(85)90037-8

POMPÉIA, Sabine; MIRANDA, Mônica Carolina; BUENO, Orlando Francisco A.A set of 400 pictures standardised for portuguese - Norms for name agreement, familiarity and visual complexity for children and adults. Arquivos de Neuro-psiquiatria, v. 59, p. 330-337, 2001. https://doi.org/10.1590/S0004-282X2001000300004

PROTOPAPAS, Athanassios. CheckVocal: A program to facilitate checking the accuracy and response time of vocal responses from DMDX. Behavior Research Methods. v. 39, n 4, p. 859-862, 2007. https://doi.org/10.3758/BF03192979

RAPP, Brenda; GOLDRICK, Mathew. Discreteness and interactivity in spoken word production. Psychological Review, v. 107, n 3, p. 460-499. https://doi.org/10.1037/0033295X.107.3.460

ROSSION, Bruno; POURTOIS, Gille. Revisiting Snodgrass and Vanderwart's object set: The role of surface detail in basic-level object recognition. Perception, v. 33, p. 217-236, 2004. https://doi.org/10.1068/p5117

SANFELIU, Maria. Carmen; FERNÁNDEZ, Angel.A set of 254 Snodgrass-Vanderwart pictures standardized for Spanish: Norms for name agreement, image agreement, familiarity, and visual complexity. Behavior Research Methods, Instruments, \& Computers, v. 28, n 4, p. 537-555, 1996. https://doi.org/10.3758/BF03200541
SCARPA, Esther M. Marcado vs. Não-marcado na aquisição e na afasia. Estudos Linguísticos, v. XXXIV, p. 839-844, 2005.

SCHWARTZ, Myrian; WILSHIRE Carolyn, E.; Gagnon, Deborah A.; Polansky Marcia. Origins of nonword phonological errors in aphasic picture naming. Cognitive Neurospychology v. 21, p. 159-186, 2004. https://doi.org/10.1080/02643290342000519

SCLIAR-CABRAL, Leonor; BARBOSA, Ana Maria Soares. Adaptação ao português do Teste M1-Alpha de André Roch Lecours e respectivo protocolo. Montreal: Guggenheim Foundation, 1981.

SENNA, Fernanda D. Acesso e representação lexical na produção de afásicos sob a ótica da Fonologia de Uso. 2013. 189 fl. Tese (Doutorado em Linguística) - Universidade Federal do Rio de Janeiro, Rio de Janeiro, 2013.

SNODGRASS, Joan G.; VANDERWART, Mary. A Standardized Set of 260 Pictures: Norms for Name Agreement, Image Agreement, Familiarity and Visual Complexity. Journal of Experimental Psychology: Human Learning and Memory. v. 6, n 2, p. 174-215, 1980. https://doi.org/10.1037/0278-7393.6.2.174

VITEVITCH, Michael S.; STAMER, Melissa K. The curious case of competition in Spanish speech production. Language and Cognitive Processes, v. 21, n 3, p. 760-770, 2006. 


\section{ANEXOS}

\section{ANEXo I}

Perfil dos voluntários pertencentes ao grupo de pacientes afásicos

\begin{tabular}{|c|c|c|c|c|c|c|c|c|c|c|c|c|c|}
\hline $\begin{array}{c}\text { Iniciais } \\
\text { Afásicos }\end{array}$ & $\begin{array}{c}\text { Sexo } \\
\text { F/M }\end{array}$ & Idade & Escolaridade & $\begin{array}{l}\text { Diagnóstico } \\
\text { Atual: Afasia }\end{array}$ & Tempo de lesão & Causa & Tempo terapia & $\begin{array}{c}\text { Mini- } \\
\text { mental }\end{array}$ & $\begin{array}{l}\text { \% pontuação } \\
\text { mini-mental }\end{array}$ & $\begin{array}{l}\text { Montreal } \\
\text { compr. } \\
\text { Oral }\end{array}$ & $\begin{array}{l}\text { \% teste } \\
\text { compreen } \\
\text { säo oral }\end{array}$ & $\begin{array}{c}\text { repeti } \\
\text { çäo }\end{array}$ & $\begin{array}{c}\text { \% teste de } \\
\text { repetição de } \\
\text { palavras reais }\end{array}$ \\
\hline S.R. & $\mathrm{F}$ & 50 & superior incompleto & Anômica & mais de 1 ano & AVE & mais de 1 ano & 28 & 100,00 & 10 & 90,91 & 8 & 72,73 \\
\hline A.A. & $\mathrm{F}$ & 84 & superior completo & Broca & mais de 4 anos & AVE & mais de 3 anos & 18 & 69,23 & 9 & 81,82 & 9 & 81,82 \\
\hline J.A. & $M$ & 55 & $2^{\circ} \mathrm{grau}$ completo & Mista & mais de 5 anos & AVE & \begin{tabular}{|l|} 
mais de 2 anos \\
\end{tabular} & 17 & 70,83 & 10 & 90,91 & 7 & 63,64 \\
\hline M.E. & $M$ & 38 & $2^{\circ}$ grau completo & Mista & mais de 5 anos & TCE & mais de 4 anos & 7 & 29,17 & 7 & 63,64 & 7 & 63,64 \\
\hline M.R. & $M$ & 25 & $2^{\circ} \mathrm{grau}$ incompleto & 1 & menos de 1 ano & TCE & menos de 1 ano & NA & & NA & & NA & \\
\hline L.B. & $\mathrm{F}$ & 59 & fundamental incompleto & Anômica & mais de 1 ano & AVE & menos de 1 ano & 8 & 38,10 & 11 & 100,00 & 8 & 72,73 \\
\hline S.S. & $\mathrm{F}$ & 62 & superior completo & Mista & mais de 3 anos & AVE & menos de 1 ano & 26 & 100,00 & 11 & 100,00 & 8 & 72,73 \\
\hline C.L. & $\mathrm{F}$ & 60 & superior completo & Mista & mais de 3 anos & 1 & mais de 1 ano & 15 & 57,69 & 11 & 100,00 & 8 & 72,73 \\
\hline P.A. & M & 51 & $2^{\circ} \mathrm{grau}$ completo & \begin{tabular}{|c|} 
Transcortical \\
Sensorial
\end{tabular} & menos de 1 ano & TCE & menos de 1 ano & 15 & 62,50 & 9 & 81,82 & 8 & 72,73 \\
\hline V.V. & $F$ & 30 & superior completo & Anômica & mais de 4 anos & AVE & mais de 2 anos & 25 & 96,15 & 11 & 100,00 & 11 & 100,00 \\
\hline M.A. & $\mathrm{F}$ & 56 & fundamental incompleto & Mista & mais de 2 anos & AVE & mais de 2 anos & 25 & 100,00 & 10 & 90,91 & 2 & 18,18 \\
\hline R.G. & $\mathrm{F}$ & 81 & analfabeta funcional & Global & menos de 1 ano & AVE & menos de 1 ano & 0 & 0,00 & 2 & 18,18 & 1 & 9,09 \\
\hline J.L. & $M$ & 76 & fundamental incompleto & Mista & menos de 1 ano & AVE & menos de 1 ano & 20 & 95,24 & 10 & 90,91 & 11 & 100,00 \\
\hline A.R & M & 66 & $2^{\circ} \mathrm{grau}$ completo & Wernicke & menos de 1 ano & AVE & menos de 1 ano & 0 & 0,00 & 3 & 27,27 & 2 & 18,18 \\
\hline G.C. & $F$ & 55 & superior completo & Mista & mais de 2 anos & 1 & menos de 1 ano & 16 & 61,54 & 9 & 81,82 & 8 & 72,73 \\
\hline T.R. & $\mathrm{F}$ & 28 & superior completo & Anômica & menos de 1 ano & AVE & menos de 1 ano & 26 & 100,00 & 11 & 100,00 & 11 & 100,00 \\
\hline C.O. & $M$ & 55 & superior incompleto & Mista & mais de 3 anos & AVE & mais de 1 ano & 24 & 92,31 & 10 & 90,91 & 10 & 90,91 \\
\hline S.M. & $M$ & 67 & $2^{\circ} \mathrm{grau}$ completo & Anômica & menos de 1 ano & AVE & menos de 1 ano & 26 & 100,00 & 11 & 100,00 & 11 & 100,00 \\
\hline F.J. & M & 51 & superior incompleto & Anômica & mais de 9 anos & AVE & mais de 4 anos & 21 & 80,77 & 8 & 72,73 & 10 & 90,91 \\
\hline F.R. & $M$ & 33 & superior incompleto & Broca & mais de 12 anos & AVE & mais de 4 anos & 20 & 76,92 & 11 & 100,00 & 9 & 81,82 \\
\hline E.T. & $M$ & 29 & superior incompleto & Broca & mais de 5 anos & TCE & mais de 3 anos & 9 & 34,62 & 8 & 72,73 & 3 & 27,27 \\
\hline M.H. & $\mathrm{F}$ & 45 & superio incompleto & Anômica & mais de 6 anos & AVE & mais de 2 anos & 23 & 88,46 & 10 & 90,91 & 8 & 72,73 \\
\hline L.F. & $\mathrm{F}$ & 39 & $2^{\circ} \mathrm{grau}$ completo & Anômica & mais de 2 anos & AVE & mais de 1 ano & 28 & 100,00 & 11 & 100,00 & 9 & 81,82 \\
\hline
\end{tabular}




\section{ANEXo II}

Perfil dos voluntários pertencentes ao grupo controle

\begin{tabular}{|c|c|c|c|}
\hline Iniciais & $\begin{array}{c}\text { Sexo } \\
\text { F/M }\end{array}$ & Idade & Escolaridade \\
\hline V.P. & $\mathrm{F}$ & 26 & $2^{\circ}$ grau completo \\
\hline B.T. & $\mathrm{M}$ & 26 & $2^{\circ}$ grau completo \\
\hline L.F. & $\mathrm{F}$ & 27 & superior completo \\
\hline V.L. & $\mathrm{F}$ & 33 & superior incompleto \\
\hline L.T. & $\mathrm{M}$ & 33 & superior incompleto \\
\hline L.B. & $\mathrm{M}$ & 35 & superior completo \\
\hline B.D. & $\mathrm{M}$ & 37 & superior completo \\
\hline E.S. & $\mathrm{F}$ & 45 & fundamental \\
\hline S.R. & $\mathrm{M}$ & 52 & superior incompleto \\
\hline E.F. & $\mathrm{F}$ & 53 & superior completo \\
\hline M.C. & $\mathrm{F}$ & 53 & $2^{\circ}$ grau incompleto \\
\hline J.O. & $\mathrm{M}$ & 54 & $2^{\circ}$ grau completo \\
\hline J.A. & $\mathrm{M}$ & 55 & superior incompleto \\
\hline L.M. & $\mathrm{F}$ & 55 & superior incompleto \\
\hline E.E. & $\mathrm{F}$ & 60 & fundamental incompleto \\
\hline M.A. & $\mathrm{M}$ & 68 & superior completo \\
\hline I.A. & $\mathrm{F}$ & 61 & fundamental completo \\
\hline L.A. & $\mathrm{M}$ & 63 & $2^{\circ}$ grau completo \\
\hline R.B. & $\mathrm{F}$ & 65 & $2^{\circ}$ grau completo \\
\hline A.G. & $\mathrm{F}$ & 65 & fundamental \\
\hline E.D. & $\mathrm{M}$ & 71 & $2^{\circ}$ grau incompleto \\
\hline N.C. & $\mathrm{F}$ & 80 & fundamental incompleto \\
\hline J.C. & $\mathrm{M}$ & 83 & fundamental \\
\hline & & & \\
\hline
\end{tabular}

Recebido em: 25/01/2017

Aprovado em: 28/06/2017 